\title{
Effect of Below Knee Prosthesis on Muscle Force Production and Joint Contact Forces of Knee and Hip Joints during Walking in Amputees
}

\section{ART ICLE INF O}

\section{Article Type}

Descriptive Study

\section{Authors}

Kamali M.*MSc,

Sharif-Moradi K. ${ }^{1} \mathrm{PhD}$,

Tahmasebi A. ${ }^{2} M S C$,

Jabal-Ameli Kh. ${ }^{3} \mathrm{PhD}$

How to cite this article
Kamali M, Sharif-Moradi K, Ta-
hmasebi A, Jabal-Ameli Kh. Eff-
ect of Below Knee Prosthesis on
Muscle Force Production and Joi-
int Contact Forces of Knee and Hip
Joints during Walking in Amp-
utees. Iranian Journal of War \&
Public Health. 2017;9(2):79-84.

*Orthoses \& Prosthesis Department, Rehabilitation Sciences Faculty, Isfahan University of Medical Sciences, Isfahan, Iran

${ }^{1}$ Physical Education \& Sport Sciences Department, Literature \& Human Sciences Faculty, Kashan University, Kashan, Iran

${ }^{2}$ Occupational Therapy Department, Rehabilitation Faculty, Tehran University of Medical Sciences, Tehran, Iran

${ }^{3}$ Orthopaedic Surgery Department, Medicine Faculty, Isfahan University of Medical Sciences, Isfahan, Iran

\section{Correspondence}

Address: Orthoses \& Prosthesis Department, Rehabilitation Sciences Faculty, Isfahan University of Medical Sciences, Hezar-Jarib Street, Isfahan, Iran

Phone: +98 (31) 12240564

Fax: -

mostafa_kamali@rehab.mui.ac.ir

\section{Article History}

Received: September 12, 2016

Accepted: January 18, 2017

ePublished: April 24, 2017

\section{A B S T R A C T}

Aims Caused by prosthesis, the kinematic and kinetic pattern changes during walking in persons with unilateral lower-limb amputation lead to muscle and joint pains. The aim of the study was to investigate the effects of below-knee prosthesis on the muscle force, as well as the knee and hip contact force, in the persons during walking.

Instrument \& Methods In the quasi-experimental study, eight non-veteran male persons with lower-knee amputation referred to Technical Orthopedic Clinic of Rehabilitation Faculty of Isfahan University of Medical Sciences were studied in 2015. The subjects were selected via available sampling method. The Qualysis motion analysis system was used to measure the walking kinematic variables. Kinematic data was recorded by Qualysis Track Manager 2.7 software and was analyzed by OpenSIM 3 software. Data was analyzed by SPSS 22 software using independent T test.

Findings The healthy stance time percentage and the healthy vibration time percentage were significantly more $(p=0.001)$ and lesser $(p=0.04)$ than the prosthetic side, respectively. The anterior-posterior, vertical, and medial-lateral hip and knee contact forces and the forces of the selected lower limbs, as well as the hip extensor, abductor, and flexor forces and the knee extensor force, at the healthy side were more than the prosthetic side, but insignificantly $(\mathrm{p}>0.05)$.

Conclusion The unilateral lower-knee prosthesis increases the healthy weight-bearing time percentage during walking. In addition, the healthy lower-limb joints are overloaded to some extent, which is due to more activities of the lower-limb muscles.

Keywords Amputee; Gait; Lower Limb, Muscles

\section{CIT A T I O N L I N KS}

[1] Limb amputation and limb deficiency: Epidemiology and recent trends in the... [2] Rehabilitation of patients after hip... [3] Asymmetrical movements of the lumbopelvic region: Is this a potential mechanism for low back pain in people with lower limb ... [4] Review of secondary physical conditions associated with lower-limb amputation ... [5] Biomechanics and physiological parameters during gait in ... [6] Adaptation strategies of the lower extremities of patients with a transtibial or transfemoral amputation during level walking ... [7] Osteoarthritis in the disabled population: A mechanical ... [8] A systematic review of psychological factors as predictors of chronicity/ disability in prospective cohorts of low back ... [9] Bio-mechanical parameters of gait among transtibial amputees ... [10] Risk factors for work-related musculoskeletal disorders: A systematic ... [11] Spinal, pelvic, and hip movement asymmetries in people with lower-limb ... [12] Persons with unilateral lower-limb amputation have altered and asymmetric trunk mechanical and neuromuscular behaviors estimated using ... [13] Three-dimensional joint reaction forces and moments at the low back during over-ground walking in persons with unilateral lower-extremity ... [14] A preliminary investigation of pelvic obliquity patterns during gait in persons with transtibial and transfemoral ... [15] Knee and hip internal moments and upper ... [16] Trunk-pelvis motion, joint loads, and muscle ... [17] Chronic low back pain in traumatic lower ... [18] Muscle power compensatory mechanisms in ... [19] Bone and joint changes in lower limb ... [20] Myofascial pain and dysfunction: The trigger point manual; The lower ... [21] Repeatability of kinematic, kinetic ... [22] OpenSim: Open-source software to create ... [23] Influence of prosthetic foot design on sound limb loading in adults with unilateral ...[24] Gait characteristics of persons with bilateral ... [25] Mechanical work adaptations of above-knee ... [26] Knee kinetics in trans-tibial ... [27] The effects of prosthetic foot roll-over shape arc length on the gait ... 
روزمره خود را بهطور مستقل انجام دهد [5]. عضو سالم با افزايش

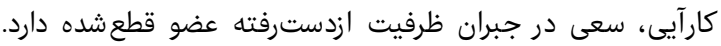

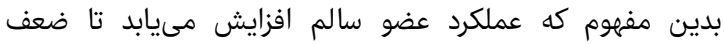

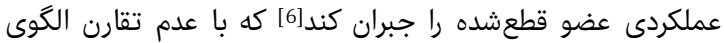

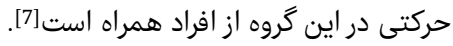

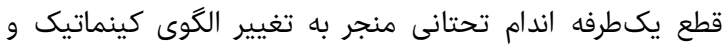

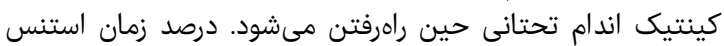

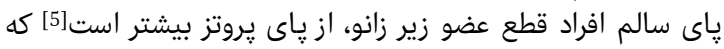

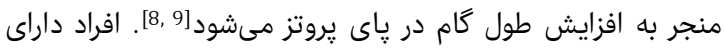

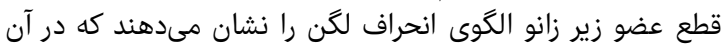

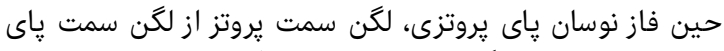

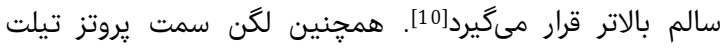

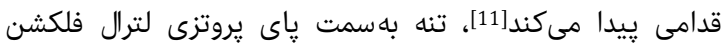

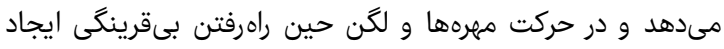

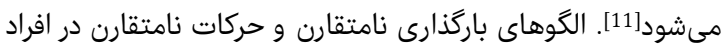

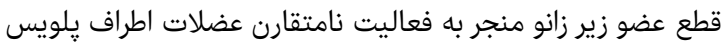

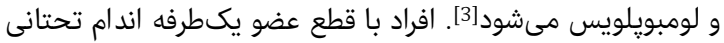

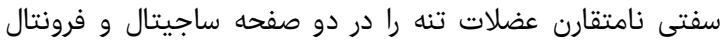

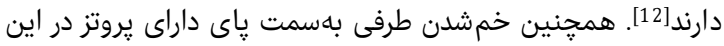

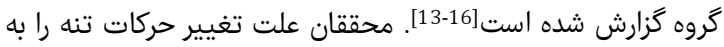

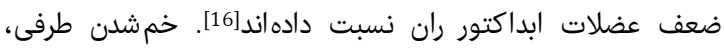

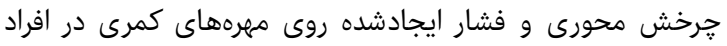

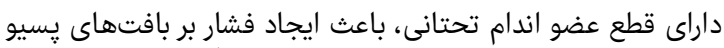

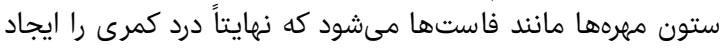

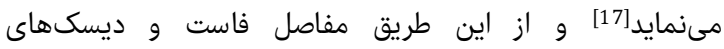

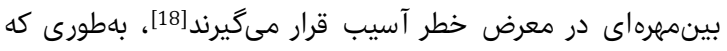

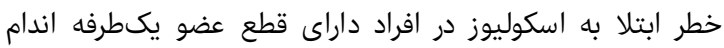

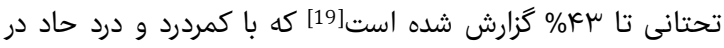

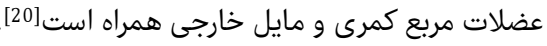

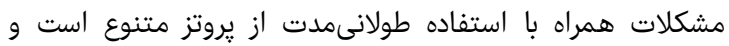

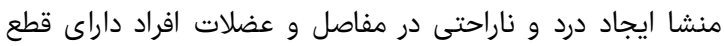

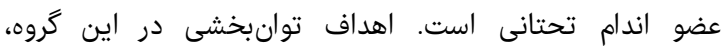

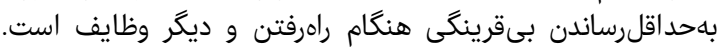

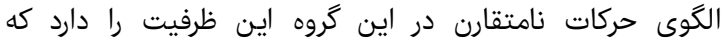

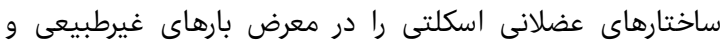

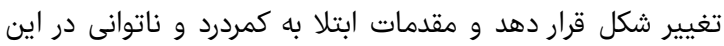

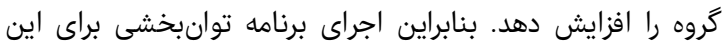

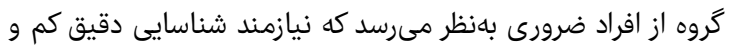
كيف تغييرات ايجادشده در سيستم عضلانى اسكلتى اين كروه ازئ دراز

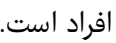

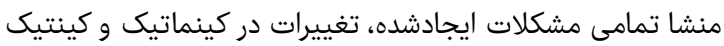

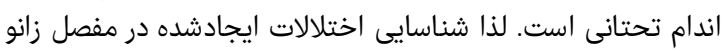

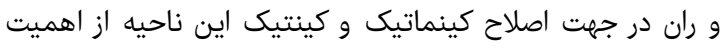

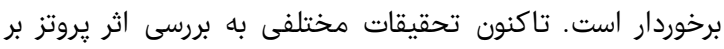

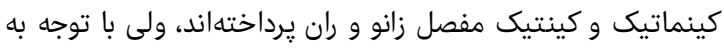

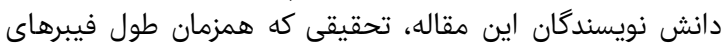

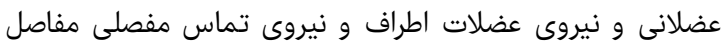

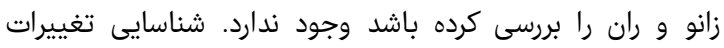

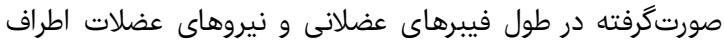

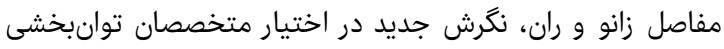

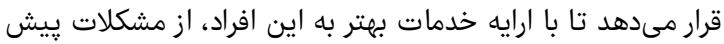

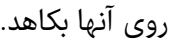

\section{تاثير يروتز زير زانو بر نيروى توليدى عضلات و

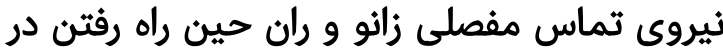 افراد با قطع عضو تماس مفصل}

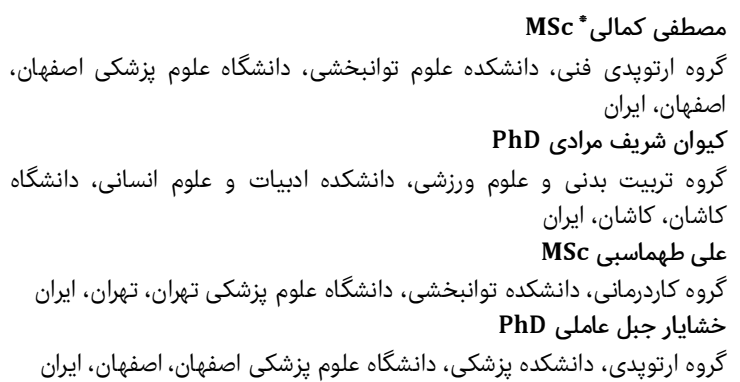

קكيده

اهداف: استفاده از يروتز در افراد با قطع عضو يكطرفيده اندام تحتانى منجر به

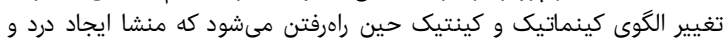

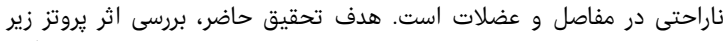

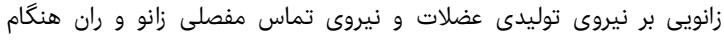

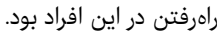

ابزار و روشها: در اين أراد بود مطالعه شبهتجربى در سال عهسا تعداد 1 مرد غيرجانباز

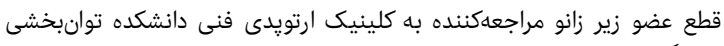

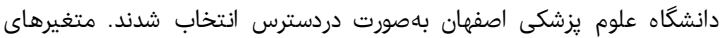

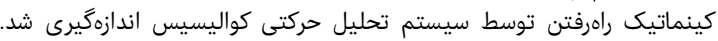
دualysis Track Manager 2.7 دادهاى كينماتي

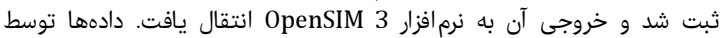

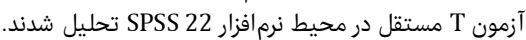

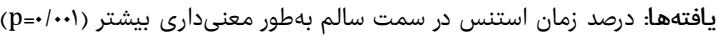

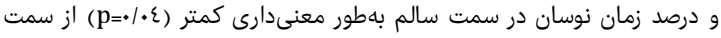

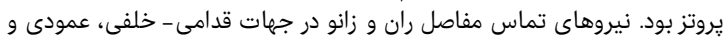

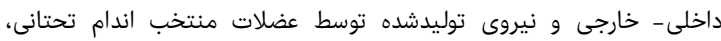

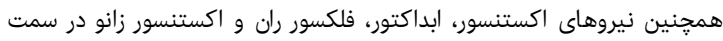

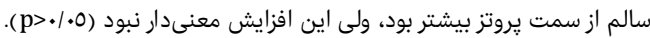

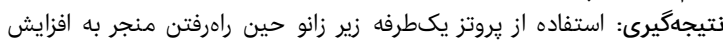

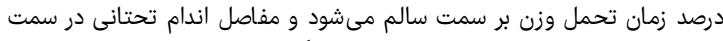

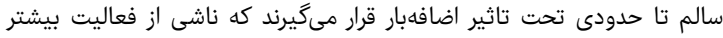

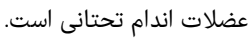
كليدوازهها: افراد با قطع عضو، ران ران رفتن، عضلات اندام تحتانى

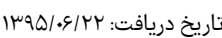

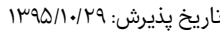

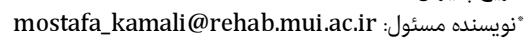

مقدمه - مقه

قطع عضو اندام تحتانى در نتيجه ضربه، تصادف، بيمارىهاى

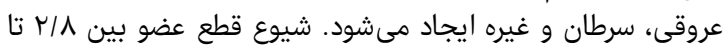

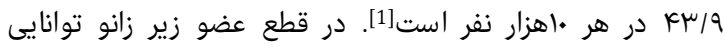

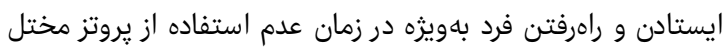

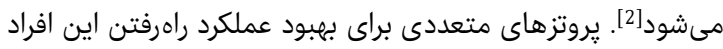

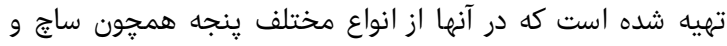

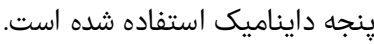

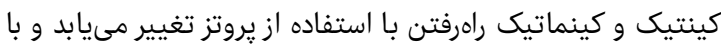
مشكلات اسكلتى عضلانى در افراد قطع عضو اندام تحتانى همراه

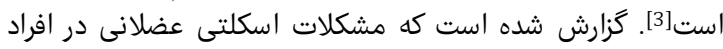

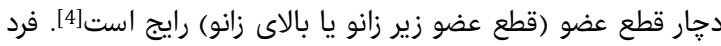

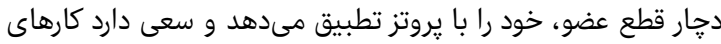




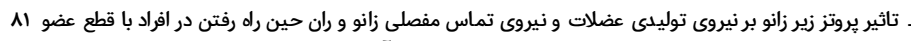

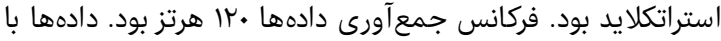

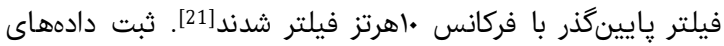

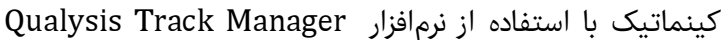
2.7 (كواليسيس؛ سوئيس) صورت

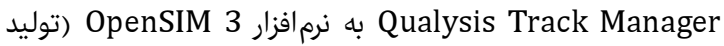
دانشكاه استانفورد؛ ايالات متحده) بهمنظور بررسى نيروى عضلات نزات

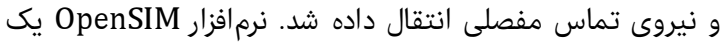

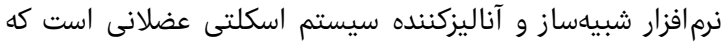

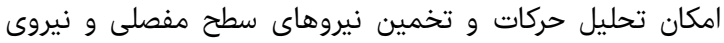

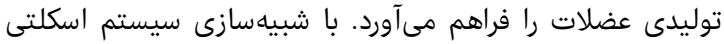

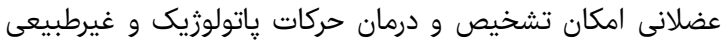

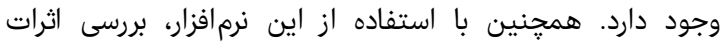

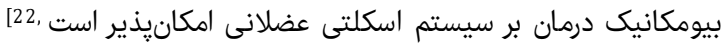

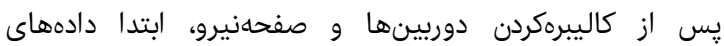

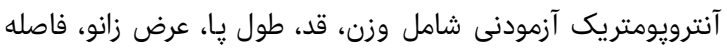

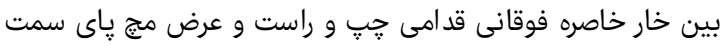

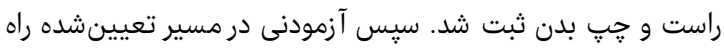

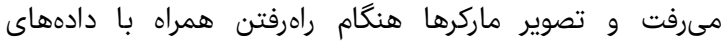

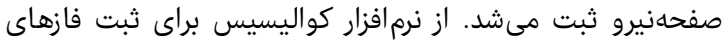

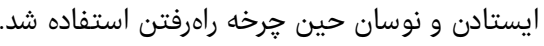

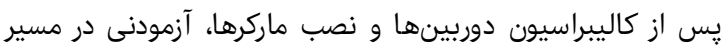

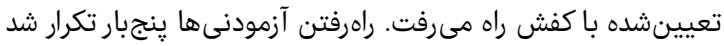

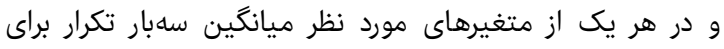

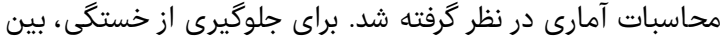

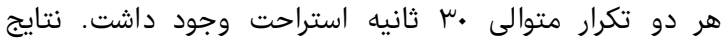

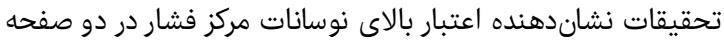

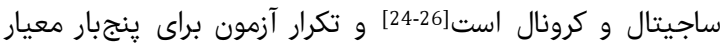

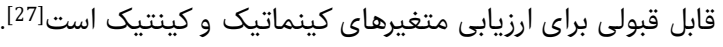

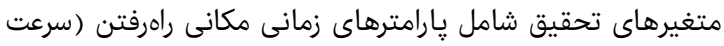

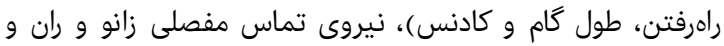

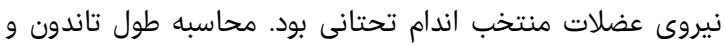

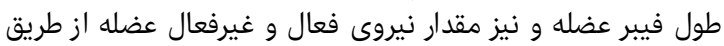

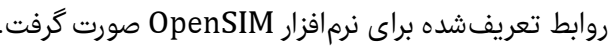

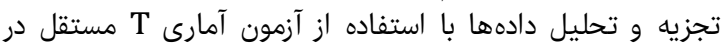

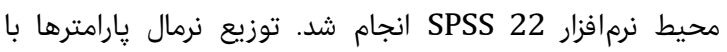

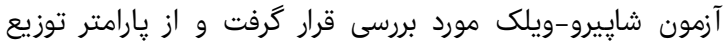

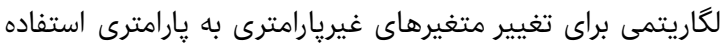

لذا هدف از تحقيق حاضر، بررسى اثر يروتز زير زانويى بر نيروى تئي

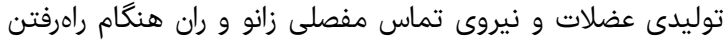
در افراد با قطع عضو اندام تحتانى بود.

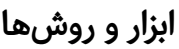

در اين مطالعه شبهتجربى در سال عوسا، تعداد •ا مرد غيرجانباز

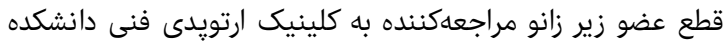

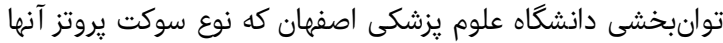

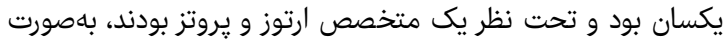

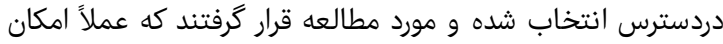

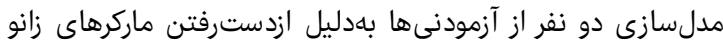

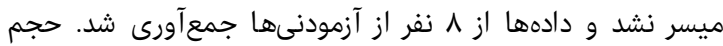

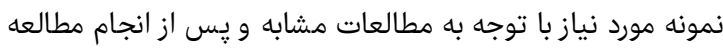

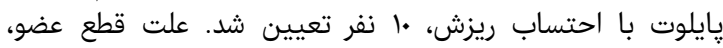

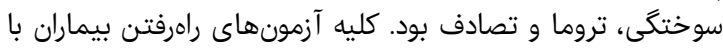

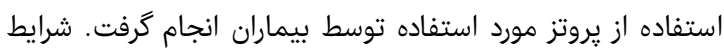

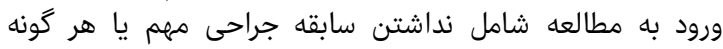

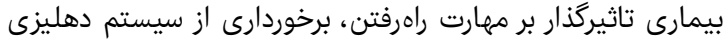

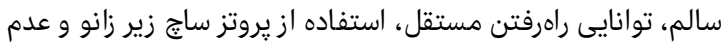

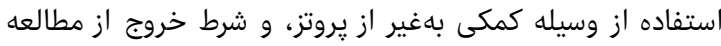

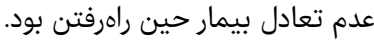

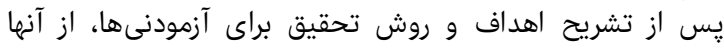

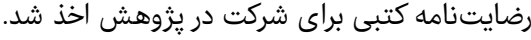

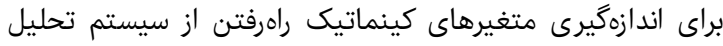

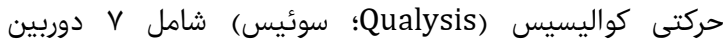

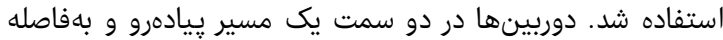

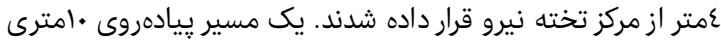

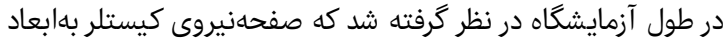

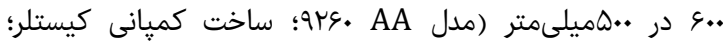

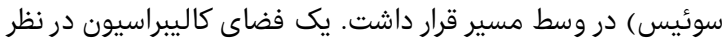

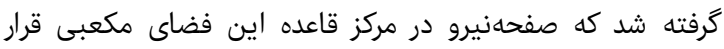
داشت. فاصله نقطه شروع راهرفتن آزمودنىها تا صفحهن دنيرو ممتر

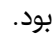
تعداد • r ماركر منعكسكننده نور مادون قرمز با قطر عاميلىمتر

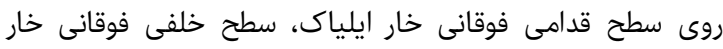

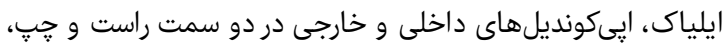

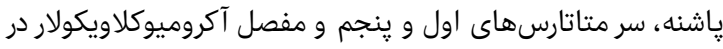

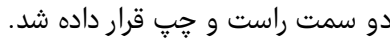
محل قرارگيرى ماركرها روى بدن براساس يرواست داده ند مصوب دانشگاه

جدول () مشخصات دموكرافيك آزمودنىها

\begin{tabular}{|c|c|c|c|c|c|c|}
\hline سمت يروتز & يروتز مورد استفاده & علت قطع عضو & قد (سانتىمتر) & وزن (كيلوگرم) & سن (سال) & شماره آزمودنى \\
\hline קו & يديلينى، با ٍنجه ساج اتوبوك & سوختگى & ivo & $\mathrm{VV} / \mathrm{A}$ & ¿० & 1 \\
\hline קֶֶ & مدولار با پنجهه سينكل اكزيس، مفصل اتوبوك، ينجه اتوبوى & 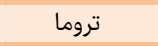 & IV & VQ/A & $\varepsilon q$ & r \\
\hline راست & 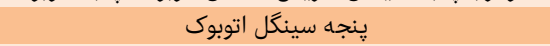 & تروما & iv. & $7 . / 4$ & $\varepsilon \wedge$ & $\mu$ \\
\hline جֶ & يروتز با پِنجه سينكل اكزيس & تروما & $17 \mathrm{~V}$ & 1.10 & $\varepsilon q$ & $\varepsilon$ \\
\hline קֶ & يروتزبا ينجه ساج وكاف استرٍ & 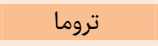 & iv. & $70 /$. & $\varepsilon V$ & 0 \\
\hline קֶ & مدولار ينجه ساج & تروما & IV乏 & $79 / \mu$ & $\varepsilon \mu$ & 7 \\
\hline 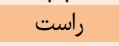 & سينكل اكزيس، اتوبوك & تروما & IV7 & $\Lambda T / \mu$ & 01 & $\checkmark$ \\
\hline 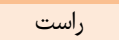 & يروتز با ينجه ساج وكاف استرٍ & تصادف & IV7 & $\mathrm{NN} / \mu$ & or & $\wedge$ \\
\hline
\end{tabular}


همجنين نيروهاى اكستنسور ران، ابداكتور ران، فلكسور ران و و

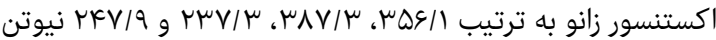

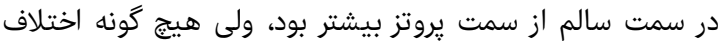

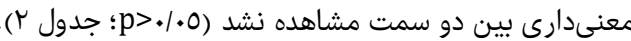

بحث

هدف از تحقيق حاضر، بررسى اثر يروتز يكطرفه زانو بر نيروى

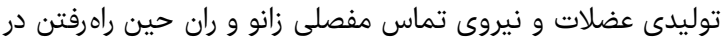

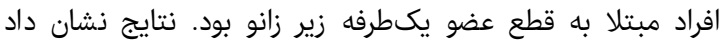

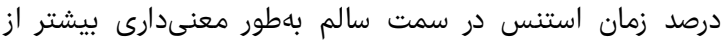

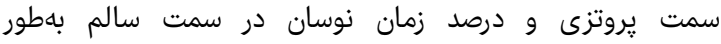

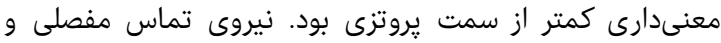

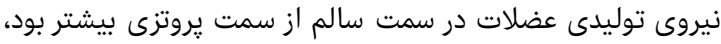
ولى اين اختلاف معنىدار نبود.

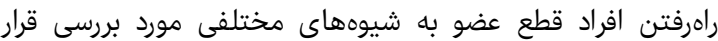

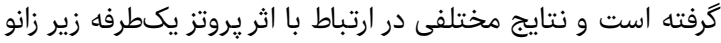

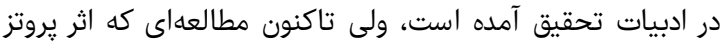

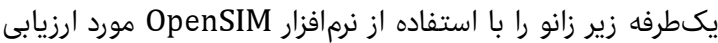

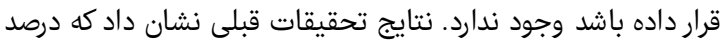

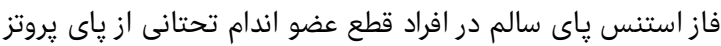

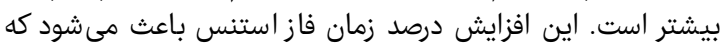

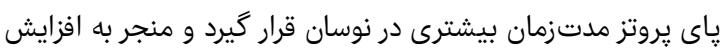

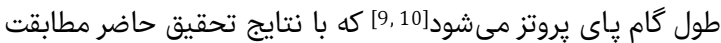

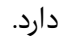

نتايج نشان داد كه يروتز يكطرفه زير زانو حين راهرفتن باعث

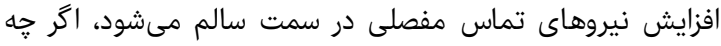

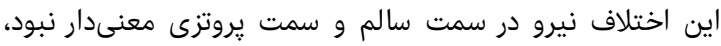

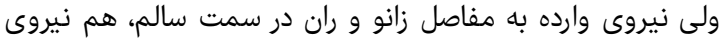

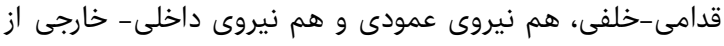

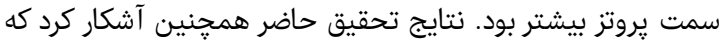

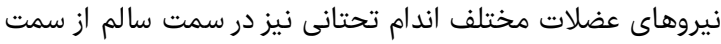

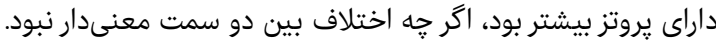

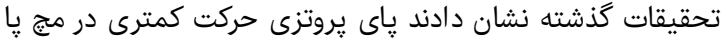

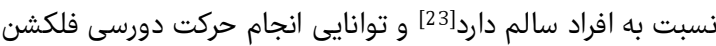

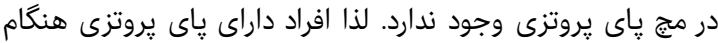

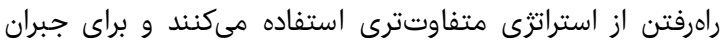

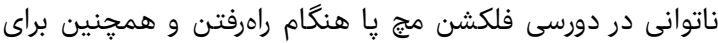

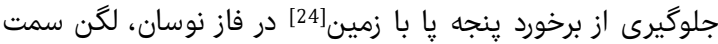

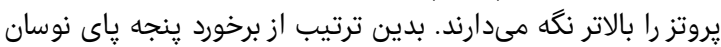

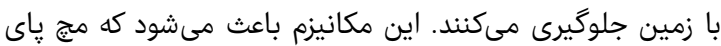

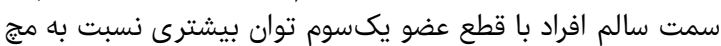

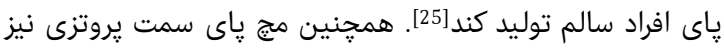

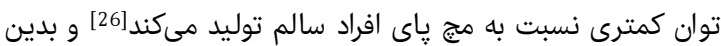

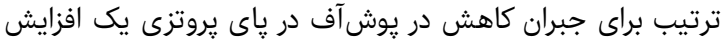

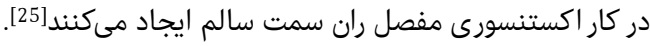

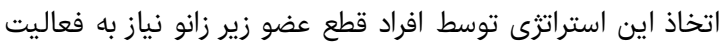

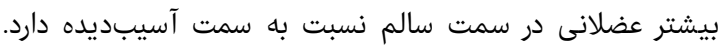

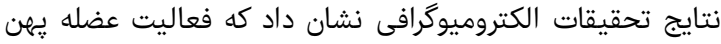

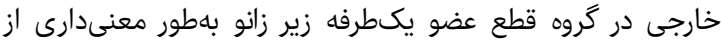

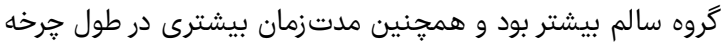

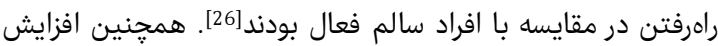

دوره 9، شماره r، بهار عوسا

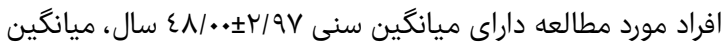

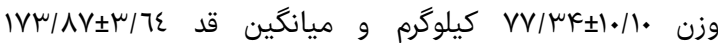

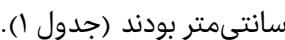

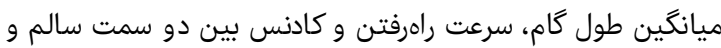

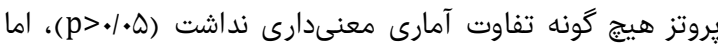

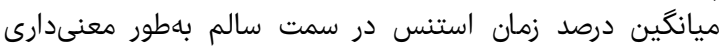

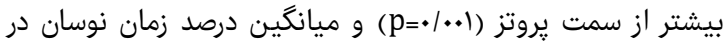

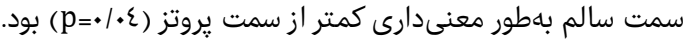

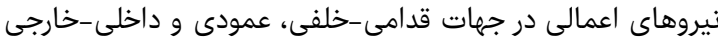

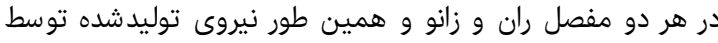

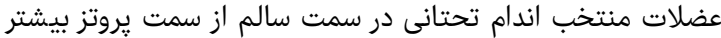

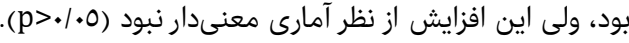

جدول r) مقايسه ميانكَين آمارى متغيرهاى يزوهش در پاى سالم و پِاى يروتز

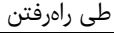

\begin{tabular}{|c|c|c|}
\hline \multicolumn{3}{|l|}{ لِياى يروتز } \\
\hline & & يارامترهاى زمانى مكانى راهرفتن \\
\hline $1 / / \gamma \pm \cdot / / \varepsilon$ & $|/ r| \pm \cdot / \mid \mu$ & طول گام (متر) \\
\hline $1 / \mu \mu_{ \pm} \cdot / 91$ & $1 / r q \pm \cdot / V r$ & 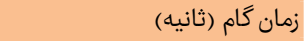 \\
\hline$\vartheta / \cdot \wedge \pm 1 / \varepsilon 0$ & $q / 1 r \pm 1 / \mu\urcorner$ & سرعت (متر بر ثانيه) \\
\hline$q \Psi / \cdot . \pm 7 / \cdot$. & $91 / \cdot \cdot \pm 7 / \cdot \cdot$ & كادنس (گام بر دقيقه) \\
\hline $7 \pi / \mu \mu \pm 1 / \lambda 7$ & $70 / 79 \pm Y / \wedge 7$ & درصد زمان استنس \\
\hline$r V / I \mu \pm r / O V$ & $\mu r / \varepsilon V \pm r / q r$ & درصد زمان نوسان \\
\hline & & نيروى تماس مفصلى (نيوتن) \\
\hline$V T \Lambda / V V \pm \varepsilon 01 / \varepsilon V$ & 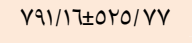 & قدامى- خلفى مفصل ران \\
\hline 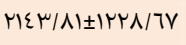 & raro/9 $\pm 17.9 / \mu \varepsilon$ & عمودى مفصل ران \\
\hline$|\varepsilon| / 7 \varepsilon \pm \varepsilon \mid / / \varepsilon$ & $\mid T \Lambda / V Y \pm \Lambda \Lambda / T 1$ & داخلى - خارجى مفصل ران \\
\hline$|\Lambda \varepsilon / r q \pm| V T / \cdot V$ & $\mu \mu o / \varepsilon V \pm 197 / \cdot v$ & قدامى - خلفى مفصل زانو \\
\hline \multicolumn{2}{|c|}{ 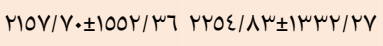 } & عمودى مفصل زانو \\
\hline$T V / / \mu_{ \pm} \mu \cdot / r$. & $1 . r / V \Psi \pm q \Psi / T V$ & داخلى - خارجى مفصل زانو \\
\hline \multicolumn{3}{|c|}{ نيروى عضلات منتخب اندام تحتانى (نيوتن) } \\
\hline $777 / V \cdot \pm 11 \varepsilon / 0$. & $V\urcorner 0 / \varepsilon \cdot \pm r \mu \cdot / r$. & كلوتئوس مديوس ا \\
\hline $1.0 / 1 \cdot \pm V 9 / r$. & $17 \varepsilon / 7 \cdot \pm 90 / 9$. & كلوتئوس مينيموس ا \\
\hline $11 \mu / \mu \cdot \pm 1 \cdot \mu / 0$. & $10 \mathrm{~N} / \mathrm{m} \cdot \pm 79 / 0$. & سمى تندينيوس \\
\hline $0 . \varepsilon / 1 \cdot \pm 110 / \mathrm{V}$ & $0 \wedge 0 / \Lambda \cdot \pm r \wedge \top / . \cdot$ & سمى ممبرانوس \\
\hline$r T \Lambda / 0 . \pm 0 N / \mu$. & $\mu / . / 9 . \pm 90 / r$. & باىسيس (سربلند) \\
\hline$\mu q \cdot 10 . \pm r \mid r / 0$. & $\varepsilon \wedge \varepsilon / V \cdot \pm r \mu \mu / \mu \cdot$ & باىسيس (سركوتاه) \\
\hline$\| \Lambda / \Lambda \cdot \pm \sum V / 1$. & $1 \cdot \mu / V \cdot \pm \mu / / 0$. & اداكتور لانكوس \\
\hline$\varepsilon \tau / r \cdot \pm r \varepsilon / r\urcorner$ & $0.17 \cdot \pm \mu / 10$ & اداكتور برويس \\
\hline$q \mu / \mu \cdot \pm r T / V$. & $1 \cdot \mu / V \cdot \pm \varepsilon 1 / \Lambda$. & اداكتور مخنوس ا \\
\hline $1 . V / 7 . \pm 0 V / 0$ & $1 \varepsilon 0 / 0 . \pm V \lambda / V$. & 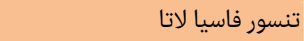 \\
\hline $1 \varepsilon q / \wedge \cdot \pm 111 / 1$. & $|\Lambda T / r \cdot \pm| r \varepsilon / Q$. & كلوتئوس ماكزيموسا \\
\hline$\varepsilon r T / 1 \cdot \pm r r O / r$. & $097 / \cdot \cdot \pm \varepsilon \mu \wedge / \mu$. & ايلياكوس \\
\hline$\varepsilon \mu \cdot / q \cdot \pm 10 \gamma / \gamma$. & $\varepsilon \varepsilon r / \varepsilon \cdot \pm r \mid q / 1$. & 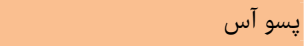 \\
\hline $1 \cdot . / 9 \cdot \pm \varepsilon \cdot / V$ & $9 \% / 7 \cdot \pm 01 / 9$. & كوادراتوس فموريس \\
\hline$r N / r \cdot \pm I r / q$. & $\mu r / r \cdot \pm I r / r$. & 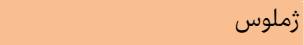 \\
\hline $179 / r \cdot \pm V / / \mu$. & $r \cdot q / 1 \cdot \pm \lambda \cdot / \Lambda$. & بيريفورميس \\
\hline $17 \varepsilon / 1 \cdot \pm \lambda \cdot / 9$. & $r \varepsilon 0 / q . \pm \mid \mu r / \varepsilon$. & يهن داخلى \\
\hline$r \varepsilon \tau / \varepsilon \cdot \pm 1 \cdot \mu / 7$. & $r \cdot 0 / V \cdot \pm 100 / 7$ & 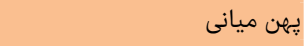 \\
\hline$\varepsilon \cdot \Lambda / 1 \cdot \pm|\Lambda| / \Lambda \cdot$ & $0 \cdot \Lambda / \mu \cdot \pm r q 0 / \mu$. & 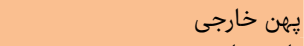 \\
\hline \multirow[t]{2}{*}{$\Lambda 7 / 0 . \pm 17 r / 7}$. & $\varepsilon q \uparrow / . . \pm|V| / \varepsilon$. & راست رانى \\
\hline & \multicolumn{2}{|c|}{ مجموع نيروهاى توليدشده در صفحات مختلف (نيوتن) } \\
\hline $\mid \pi \cdot 0 / \cdot . \pm \varepsilon 70 / 0$ & $100 r / 9 \cdot \pm 700 / \mathrm{V}$ & اكستنسور زانو \\
\hline $19 Y \varepsilon / V \cdot \pm \varepsilon \varepsilon 7 / 0 \Lambda$ & $r r \Lambda \cdot / V \cdot \pm V \varepsilon I / \varepsilon \cdot$ & اكستنسور ران \\
\hline$|V| \varepsilon / \Lambda \cdot \pm \varepsilon \varepsilon \tau / \Lambda$. & $r r \Lambda \cdot / V \cdot \pm V \varepsilon / / \varepsilon O$ & 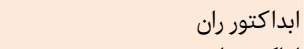 \\
\hline$\mu r \tau / \varepsilon \cdot \pm 11 r / \mu$. & rro/r. \pm 1 ro/l. & اداكتور ران \\
\hline $1 . \varepsilon 0 / \varepsilon \cdot \pm \varepsilon r \varepsilon / 1$. & $|r A Y / \Psi \cdot \pm T V| / q$. & فلكسور ران \\
\hline
\end{tabular}

فصل امه علمى - يُروهشى طب جانباز 
تاثير يروتز زير زانو برنيروى توليدى عضلات و نيروى تماس مفصلى زانو و ران حين راه رفتن در افراد با قطع عضو سرم

2- Dénes Z, Till A. Rehabilitation of patients after hip disarticulation. Arch Orthop Trauma Surg. 1997;116(8):498-9.

3- Devan H, Hendrick P, Ribeiro DC, Hale LA, Carman A. Asymmetrical movements of the lumbopelvic region: Is this a potential mechanism for low back pain in people with lower limb amputation?. Med Hypotheses. 2014;82(1):77-85.

4- Gailey R, Allen K, Castles I, Kucharik J, Roeder M. Review of secondary physical conditions associated with lower-limb amputation and long-term prosthesis use. J Rehabil Res Dev. 2008;45(1):15-29.

5- Sagawa Y Jr, Turcot K, Armand S, Thevenon A Vuillerme N, Watelain E. Biomechanics and physiological parameters during gait in lower-limb amputees: A systematic review. Gait Posture, 2011;33(4): 511-26.

6- Prinsen EC, Nederhand MJ, Rietman JS. Adaptation strategies of the lower extremities of patients with a transtibial or transfemoral amputation during level walking: A systematic review. Arch Phys Med Rehabil. 2011;92(8):1311-25.

7- Morgenroth DC, Gellhorn AC, Suri P. Osteoarthritis in the disabled population: A mechanical perspective. PM R. 2012;4(5 Suppl):S20-7.

8- Pincus T, Burton AK, Vogel S, Field AP. A systematic review of psychological factors as predictors of chronicity/disability in prospective cohorts of low back pain. Spine (Phila Pa 1976). 2002;27(5):E109-20.

9- Soares AS, Yamaguti EY, Mochizuki L, Amadio AC, Serrão JC. Biomechanical parameters of gait among transtibial amputees: A review. Sao Paulo Med J. 2009;127(5):302-9.

10- da Costa BR, Vieira ER. Risk factors for work-related musculoskeletal disorders: A systematic review of recent longitudinal studies. Am J Ind Med. 2010;53(3):285-323.

11- Devan H1, Carman A, Hendrick P, Hale L, Ribeiro DC. Spinal, pelvic, and hip movement asymmetries in people with lower-limb amputation: Systematic review. J Rehabil Res Dev. 2015;52(1):1-19.

12- Hendershot BD, Bazrgari B, Nussbaum MA. Persons with unilateral lower-limb amputation have altered and asymmetric trunk mechanical and neuromuscular behaviors estimated using multidirectional trunk perturbations. J Biomech. 2013;46(11):1907-12.

13- Hendershot BD, Wolf EJ. Three-dimensional joint reaction forces and moments at the low back during over-ground walking in persons with unilateral lowerextremity amputation. Clin Biomech (Bristol, Avon). 2014;29(3):235-42.

14- Michaud SB, Gard SA, Childress DS. A preliminary investigation of pelvic obliquity patterns during gait in persons with transtibial and transfemoral amputation. J Rehabil Res Dev. 2000;37(1):1-10.

15- Molina Rueda F, Alguacil Diego IM, Molero Sánchez A, Carratalá Tejada M, Rivas Montero FM, Miangolarra Page JC. Knee and hip internal moments and upper-body kinematics in the frontal plane in unilateral transtibial amputees. Gait Posture. 2013;37(3):436-9.

16- Yoder AJ, Petrella AJ, Silverman AK. Trunk-pelvis motion, joint loads, and muscle forces during walking with a transtibial amputation. Gait Posture. 2015;41(3):757-62.

17- Kulkarni J, Gaine WJ, Buckley JG, Rankine JJ, Adams J. Chronic low back pain in traumatic lower limb amputees. Clin Rehabil. 2005;19(1):81-6.
توان اكستنسور ران در افراد قطع عضو يكطرفه زير زانو را به به برني

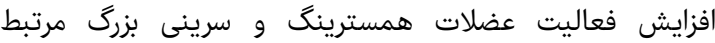

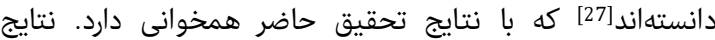

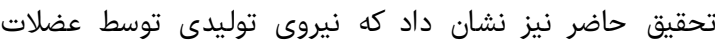

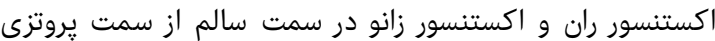

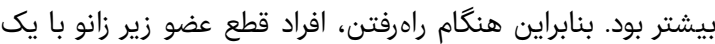

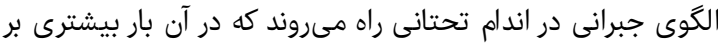

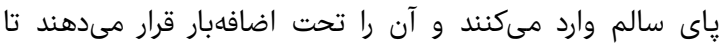
بتوانند نقص ناشى از قطع عضو را را جبران كنند.

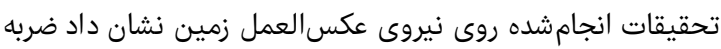

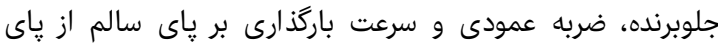

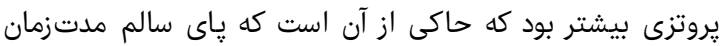

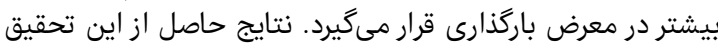

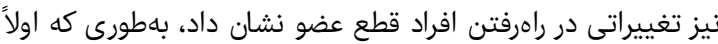

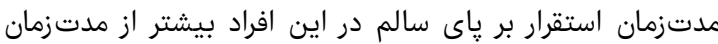

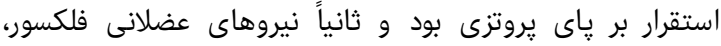

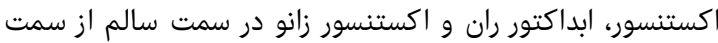

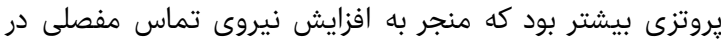

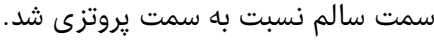

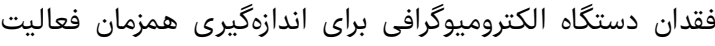

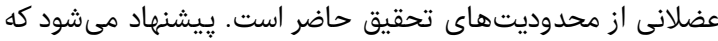

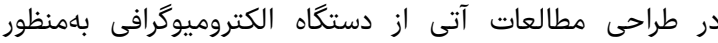
اندازمكيرى همزمان فعاليت عضلانى استفاده شود.

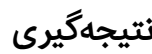

استفاده از يروتز يكطرفه زير زانو حين راهرفت ريتن منجر به افزايش

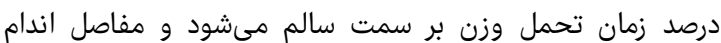

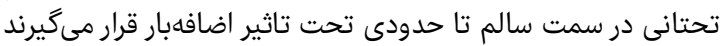
كه ناشى از فعاليت بيشتر عضلات اندام تحتانى است.

تشكر و قدردانى: بدين وسيله نويسندكان مقاله از مركز تحقيقات

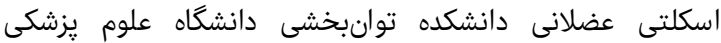

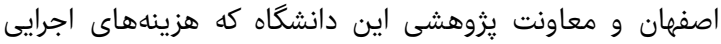

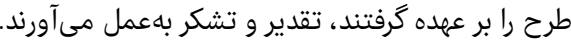

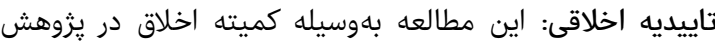

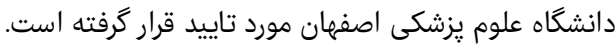

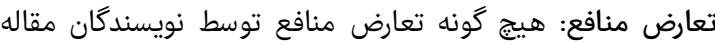
كزارش نشده است.

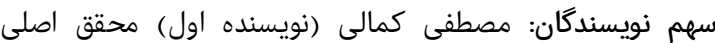

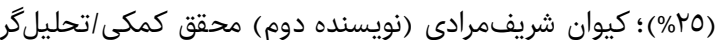

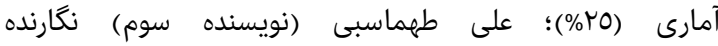

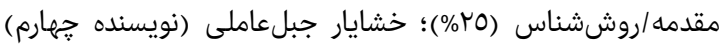

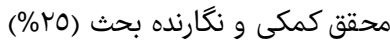

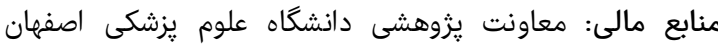
هزينههاى اجرايى اين طرح را بر عهده كرفت.

منابع 1- Dillingham TR, Pezzin LE, MacKenzie EJ. Limb amputation and limb deficiency: Epidemiology and recent trends in the United States. South Med J. 2002;95(8):875-83. 
23- Powers CM, Torburn L, Perry J, Ayyappa E. Influence of prosthetic foot design on sound limb loading in adults with unilateral below-knee amputations. Arch Phys Med Rehabil. 1994;75(7):825-9.

24- Su PF, Gard SA, Lipschutz RD, Kuiken TA. Gait characteristics of persons with bilateral transtibial amputations. J Rehabil Res Dev. 2007;44(4):491-501.

25- Seroussi RE, Gitter A, Czerniecki JM, Weaver K. Mechanical work adaptations of above-knee amputee ambulation. Arch Phys Med Rehabil. 1996;77(11):120914.

26- Powers CM, Rao S, Perry J. Knee kinetics in transtibial amputee gait. Gait Posture. 1998;8(1):1-7.

27- Hansen AH, Meier MR, Sessoms PH, Childress DS. The effects of prosthetic foot roll-over shape arc length on the gait of trans-tibial prosthesis users. Prosthet Orthot Int. 2006;30(3):286-99.
18- Sadeghi H, Allard P, Duhaime PM. Muscle power compensatory mechanisms in below-knee amputee gait. Am J Phys Med Rehabil. 2001;80(1):25-32.

19- Burke MJ, Roman V, V Wright. Bone and joint changes in lower limb amputees. Ann Rheum Dis. 1978; 37(3): 252-254.

20- Lang Newman DI. Myofascial pain and dysfunction: The trigger point manual; The lower extremities. Clin J Pain. 1992;8(2):178.

21- Kadaba MP, Ramakrishnan HK, Wootten ME, Gainey J, Gorton G, Cochran GV. Repeatability of kinematic, kinetic, and electromyographic data in normal adult gait. J Orthop Res. 1989;7(6):849-60.

22- Delp SL, Anderson FC, Arnold AS, Loan P, Habib A, John CT, et al. OpenSim: Open-source software to create and analyze dynamic simulations of movement. IEEE Trans Biomed Eng. 2007;54(11):1940-50. 